\title{
14. SECULAR PERTURBATIONS ON PERIODIC COMETS
}

\author{
G. E. O. GIACAGLIA \\ Dept. of Aerospace Engineering and Engineering Mechanics, \\ University of Texas, Austin, Tex., U.S.A.*
}

\begin{abstract}
In this work we propose an approach to the calculation of secular perturbations on a comet, valid for any eccentricity (less than unity) and any inclination (not zero). The effect of several planets is discussed, but we suppose that there are no significant close approaches to any planet.
\end{abstract}

\section{Disturbing Function}

The force function due to a planet - considered to be moving in a circular orbit around the Sun - corresponds in the secular sense to the force exerted upon the comet by a loaded ring. It is therefore necessary to obtain the potential of such a ring, giving an expression valid for distances less than or greater than the radius of the ring. The classical development in spherical harmonics has therefore to be abandoned.

According to Figure 1, let $a^{\prime}$ be the radius of the ring, $\delta$ the latitude of the comet $P$, $\alpha$ its longitude, $r$ its distance from the Sun (center of the ring), $\Delta$ the distance between $P$ and $P^{\prime}$ (a point on the ring with longitude $\alpha^{\prime}$ ) and $\psi$ their mutual elongation.

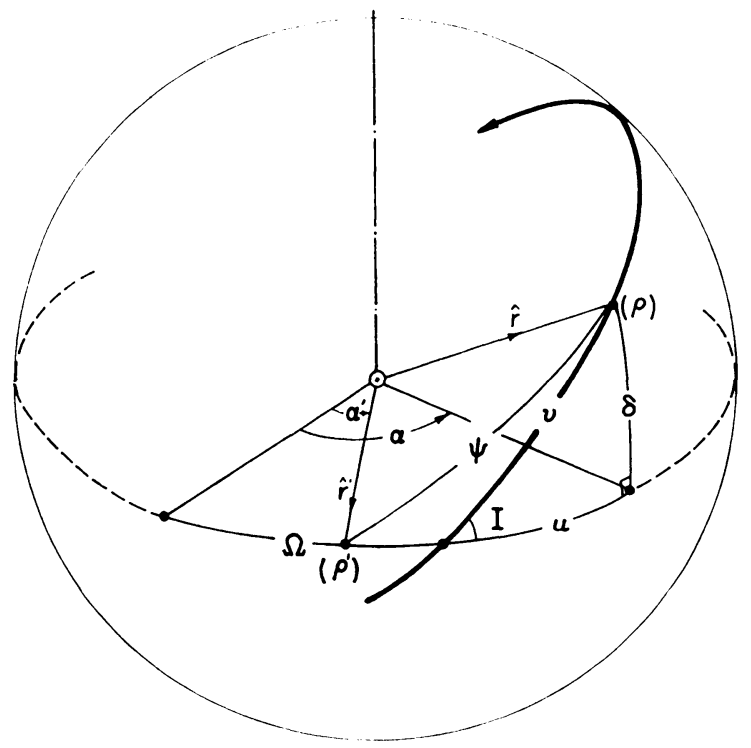

Fig. 1. Geometry of the problem.

* On leave from the University of São Paulo and University of Campinas, São Paulo, Brazil. 
It follows that

$$
\begin{aligned}
\cos \psi & =\cos \delta \cos \left(\alpha^{\prime}-\alpha\right) \\
\Delta^{2} & =r^{2}+a^{2}-2 a^{\prime} r \cos \psi
\end{aligned}
$$

and, using osculating elements,

$$
r=a(1-e \cos E),
$$

where $E$ is the eccentric anomaly of the comet, $a$ the semimajor axis and $e$ the eccentricity. The maximum value of $\Delta$ is, at any instant,

$$
A=a^{\prime}+a(1+e),
$$

so that we write

$$
\Delta^{2}=A^{2}\left\{1-\left[B+C \cos \left(\alpha^{\prime}-\alpha\right)\right] / A^{2}\right\},
$$

where

$$
\begin{aligned}
B & =\rho-r^{2} \\
\rho & =a(1+e)\left[a(1+e)+2 a^{\prime}\right] \\
C & =2 a^{\prime} r \cos \delta .
\end{aligned}
$$

Evidently, unless $\Delta=0$, the expansion by the binomial formula of $\Delta^{-1}$ will converge for all values of $r$, so that one can write the series

$$
\Delta^{-1}=A^{-1} \sum_{k=0}^{\infty}\left(\begin{array}{c}
-\frac{1}{2} \\
k
\end{array}\right)(-1)^{k} A^{-2 k}\left[B+C \cos \left(\alpha^{\prime}-\alpha\right)\right]^{k}
$$

or

$$
\Delta^{-1}=A^{-1} \sum_{k=0}^{\infty} \sum_{j=0}^{k}\left(\begin{array}{c}
-\frac{1}{2} \\
k
\end{array}\right)\left(\begin{array}{c}
k \\
j
\end{array}\right)(-1)^{k} A^{-2 k} B^{k-j} C^{j} \cos ^{j}\left(\alpha^{\prime}-\alpha\right)
$$

The force function of the loaded ring (mass $m^{\prime}$ ) is given by

$$
U=\int_{0}^{2 \pi} G \Delta^{-1} \lambda^{\prime} a^{\prime} \mathrm{d}\left(\alpha^{\prime}-\alpha\right),
$$

where $\lambda^{\prime}=m^{\prime} / 2 \pi a^{\prime}$ and $G$ is the gravitational constant.

Since now

$$
\begin{aligned}
& \int_{0}^{2 \pi} \cos ^{2 j+1} x \mathrm{~d} x=0 \\
& \int_{0}^{2 \pi} \cos ^{2 j} x \mathrm{~d} x=2 \pi \frac{(2 j-1) ! !}{2^{j} j !}
\end{aligned}
$$


it follows that

$$
U=\frac{G m^{\prime}}{A} \sum_{k=0}^{\infty} \sum_{j=0}^{[k / 2]}(-1)^{k}\left(\begin{array}{c}
-\frac{1}{2} \\
k
\end{array}\right)\left(\begin{array}{l}
k \\
2 j
\end{array}\right) \frac{(2 j-1) ! !}{2^{j} j !} A^{-2 k} B^{k-2 j} C^{2 j},
$$

where $[k / 2]$ is the integer part of $k / 2$.

Now let $v=\omega+f$ be the argument of the latitude ( $\omega$ the argument of perihelion, $f$ the true anomaly) and $\Omega$ the longitude of the ascending node. We have the relations

$$
\begin{aligned}
& \cos \delta \cos (\alpha-\Omega)=\cos v \\
& \cos \delta \sin (\alpha-\Omega)=\sin v \cos I,
\end{aligned}
$$

where $I$ is the inclination of the osculating orbit. Therefore

$$
C^{2 j}=4^{j} a^{2 j} r^{2 j}\left(\cos ^{2} I+\sin ^{2} I \cos ^{2} v\right)^{j},
$$

and also

$$
B^{k-2 j}=\left(\rho-r^{2}\right)^{k-2 j}=\sum_{p=0}^{k-2 j}\left(\begin{array}{c}
k-2 j \\
p
\end{array}\right)(-1)^{p} \rho^{k-2 j-p} r^{2 p},
$$

so that one finds

$$
\begin{aligned}
U= & \frac{G m^{\prime}}{A} \sum_{k=0}^{\infty} \sum_{j=0}^{[k / 2]} \sum_{p=0}^{k-2 j} \sum_{s=0}^{j} K_{k j p s}^{*} A^{-2 k} \rho^{k-2 j-p} a^{\prime 21} r^{2(p+j)} \\
& \times \cos ^{2(j-s)} I \sin ^{2 s} I \cos ^{2 s} v
\end{aligned}
$$

where

$$
K_{k j p s}^{*}=\frac{(-1)^{p}(2 k) !(2 j+1)}{4^{k} k ! p !(k-2 j-p) ! j ! s !(j-s) !} .
$$

We change the index notation by setting

$$
p+j=q, \quad K_{k j p s}^{*}=K_{k j, q-j, s}^{*}=K_{k j q s}
$$

so that

$$
\begin{aligned}
U= & \frac{G m^{\prime}}{A} \sum_{k=0}^{\infty} \sum_{j=0}^{[k / 2]} \sum_{q=j}^{k-j} \sum_{s=0}^{j} K_{k j q s} A^{-2 k} \rho^{k-j-q} a^{2 j} \\
& \times \cos ^{2(j-s)} I \sin ^{2 s} I r^{2 q} \cos ^{2 s} v .
\end{aligned}
$$

The long period and secular perturbations on the comet are generated by the average $U$ over the mean anomaly of the comet, that is

$$
U_{s}=\frac{1}{2 \pi} \int_{0}^{2 \pi} U \mathrm{~d} l,
$$

assuming, of course, no commensurability exists between the mean motion of the comet and that of the planet. It has long been known, for example, that the ratio of the periods of P/Encke and Jupiter is very close to 5/18 (Whipple, 1940). Although of very high degree, this commensurability may produce a large coefficient in the perturbations because of the excessive eccentricity of the comet (about 0.85). On the 
other hand, no attempt has been made to determine the average of $U$ analytically because, in the classical sense, it would require an expansion in powers of $e$, making any results invalid. Brouwer (1947) did obtain a good approximation to the secular variations of $\mathbf{P} /$ Encke by evaluating a numerical average of the disturbing function over the period of the system (five revolutions of Jupiter), and he obtained good agreement with Whipple's empirical results. This indicates that, at least in this case, Jupiter perturbations would suffice to give a good first approximation for the orbit.

The force function $U$ we have obtained is valid everywhere except on the ring itself - which would imply the possibility of collision. The excluded cases are therefore those which correspond to the condition $\Delta=0$ or

$$
r=a^{\prime}, \quad \psi=0
$$

or

$$
r=a^{\prime}, \quad \delta=0, \quad \alpha=\alpha^{\prime} .
$$

For the ring potential this reduces to

$$
r=a^{\prime}, \quad \delta=0 .
$$

The first of these gives the equation

$$
1-e \cos E=a^{\prime} / a,
$$

which can be satisfied in the cases when

$$
\left|1-a^{\prime}\right| a \mid \leq e \text {. }
$$

\section{Average Disturbing Function}

Next, in order to avoid any expansion in powers of the eccentricity, the function $U_{s}$ will be obtained using the eccentric anomaly as independent variable.

Let

$$
Q_{2 q, 2 s}=\frac{1}{2 \pi} \int_{0}^{2 \pi} r^{2 q} \cos ^{2 s}(f+\omega) \mathrm{d} l,
$$

where

$$
\begin{aligned}
q & =0,1,2, \ldots, k-2 j \\
s & =0,1,2, \ldots, j \\
j & =0,1, \ldots,[k / 2] \\
k & =0,1,2, \ldots
\end{aligned}
$$

Considering the relation

$$
\begin{aligned}
& \mathrm{d} l=\frac{r}{a} \mathrm{~d} E, \\
& Q_{2 q, 2 s}=\frac{a^{2 q}}{2 \pi} \int_{0}^{2 \pi}\left(\frac{r}{a}\right)^{2 q+1} \cos ^{2 s}(f+\omega) \mathrm{d} E .
\end{aligned}
$$


If we let $\varepsilon=\exp (i E)$, we find that

$$
\begin{aligned}
\left(\frac{r}{a}\right)^{2 q+1} \cos ^{2 s} v= & \frac{1}{2^{2 s}} \sum_{j=0}^{2 s} \sum_{k=0}^{2(s-j) k+2 q+1} \sum_{\gamma=k}^{k} \sum_{\alpha=0}^{k} \sum_{\beta=0}^{\gamma-k} L_{j, k, \gamma, \alpha, \beta}^{2 q, 2 s} \\
& \times e^{2(s-k-j)+\gamma}(1-\eta)^{k-\alpha}(1+\eta)^{\alpha} \\
& \times \exp [-2 i(s-j) \omega] \varepsilon^{\gamma-2(\alpha+\beta)}
\end{aligned}
$$

where $\eta=\sqrt{1-e^{2}}$ and

$$
L_{j, k, \gamma, \alpha, \beta}^{2 q, 2 s}=(-2)^{-\gamma}\left(\begin{array}{c}
2 s \\
j
\end{array}\right)\left(\begin{array}{c}
2 s-2 j \\
k
\end{array}\right)\left(\begin{array}{c}
2 q+1 \\
\gamma-k
\end{array}\right)\left(\begin{array}{c}
k \\
\alpha
\end{array}\right)\left(\begin{array}{c}
\gamma-k \\
\beta
\end{array}\right) .
$$

Contributions to the integral $Q_{2 q, 2 s}$ come from even values of $\gamma$, so that the corresponding terms are

$$
\begin{aligned}
{\left[\left(\frac{r}{a}\right)^{2 q+1} \cos ^{2 s} v\right]_{\mathrm{even}}=} & \frac{1}{2^{2 s}} \sum_{j=0}^{2 s} \sum_{k=0}^{2(s-i)[(k+1+2 q) / 2]} \sum_{\gamma=[(k+1) / 2]}^{h} \sum_{\alpha=0}^{2 \gamma-k} \sum_{\beta=0}^{2 q} L_{j, k, 2 \gamma, \alpha, \beta}^{2 q, 2 s} \\
& \times e^{2(s-k-j+\gamma)}(1-\eta)^{k-\alpha}(1+\eta)^{\alpha} \\
& \times \exp [-2 i(s-j) \omega] \varepsilon^{2(\gamma-\alpha-\beta)},
\end{aligned}
$$

and therefore, setting $\gamma=\alpha+\beta$,

$$
\begin{aligned}
2^{2 s} Q_{2 q, 2 s}= & a^{2 q} \sum_{j=0}^{2 s} \sum_{k=0}^{2(s-j)} \sum_{\gamma=[(k+1) / 2]}^{[(k+1+2 q) / 2]} \sum_{\alpha=0}^{k} L_{j, k, 2 \gamma, \alpha, \gamma-\alpha}^{2 q, 2 s} \\
& \times e^{2(s-k-j+\gamma)}(1-\eta)^{k-\alpha}(1+\eta)^{\alpha} \exp [-2 i(s-j) \omega] \\
= & 2^{2 s} a^{2 q} \sum_{\beta=0}^{s} M_{2 q, 2 s}^{\beta} \cos 2 \beta \omega,
\end{aligned}
$$

where

$$
2^{2 s} M_{2 q, 2 s}^{0}=a^{2 q} \sum_{\gamma=0}^{q} L_{s, 0,2 \gamma, 0, \gamma}^{2 q, 2 s} e^{2 \gamma}
$$

and, for $\beta \neq 0$,

$$
\begin{aligned}
M_{2 q, 2 s}^{\beta}= & \frac{1}{2^{2 s-1}} \sum_{k=0}^{2 \beta} \sum_{\gamma=[(k+1) / 2]}^{[(k+1+2 q) / 2]} \sum_{\alpha=0}^{k} L_{s-\beta, k, 2 \gamma, \alpha, \gamma-\alpha}^{2 q, 2 s} \\
& \times e^{2(\beta-k+\gamma)}(1-\eta)^{k-\alpha}(1+\eta)^{\alpha} .
\end{aligned}
$$

We finally arrive at the average disturbing function $U_{s}$, given by

where

$$
\begin{aligned}
U_{s}= & \frac{G m^{\prime}}{A} \sum_{k=0}^{\infty} \sum_{j=0}^{[k / 2]} \sum_{q=j}^{k-j} \sum_{s=0}^{j} \sum_{\beta=0}^{s} N_{k j q s}^{\beta} A^{-2 k} \\
& \times \rho^{k-j-q} a^{\prime 2 j} a^{2 q} \cos ^{2(j-s)} I \sin ^{2 s} I \cos 2 \beta \omega,
\end{aligned}
$$

$$
N_{k j, 2 q, 2 s}^{\beta}=K_{k j q s} M_{24,2 s}^{\beta},
$$


the factors on the right-hand side being defined in Equations (8), (9) and (16).

We also remember that

$$
A=a^{\prime}+a(1+e), \quad \rho=\left(A-a^{\prime}\right)\left(A+a^{\prime}\right)=A^{2}-a^{\prime 2} .
$$

\section{An Example}

As an example we develop $U_{s}$ up to $k=4$. Rearranging terms properly, we find

$$
\begin{aligned}
& U_{s}=\frac{\mu^{\prime}}{A}\left\{\left(1+\frac{1}{2} \frac{\rho}{A^{2}}+\frac{3}{8} \frac{\rho^{2}}{A^{4}}+\frac{5}{16} \frac{\rho^{3}}{A^{6}}+\frac{35}{128} \frac{\rho^{4}}{A^{8}}\right)\right. \\
& -\frac{1}{2} \frac{a^{2}}{A^{2}}\left(1+\frac{3}{2} \frac{\rho}{A^{2}}+\frac{15}{8} \frac{\rho^{2}}{A^{4}}+\frac{35}{16} \frac{\rho^{3}}{A^{6}}\right)\left(1+\frac{3}{2} e^{2}\right) \\
& +\frac{3}{8} \frac{a^{4}}{A^{4}}\left(1+\frac{5}{2} \frac{\rho}{A^{2}}+\frac{35}{8} \frac{\rho^{2}}{A^{4}}\right)\left(1+5 e^{2}+\frac{5}{16} e^{4}\right) \\
& -\frac{5}{16} \frac{a^{6}}{A^{6}}\left(1+\frac{7}{2} \frac{\rho}{A^{2}}\right)\left(1+\frac{21}{2} e^{2}+\frac{105}{8} e^{4}+\frac{35}{16} e^{6}\right) \\
& +\frac{35}{128} \frac{a^{8}}{A^{8}}\left(1-18 e^{2}+\frac{189}{4} e^{4}+\frac{105}{4} e^{6}+\frac{315}{128} e^{8}\right) \\
& +\frac{3}{4} \frac{a^{2} a^{\prime 2}}{A^{4}}\left(1+\frac{5}{2} \frac{\rho}{A^{2}}+\frac{35}{8} \frac{\rho^{2}}{A^{4}}\right)\left[\left(1+\frac{3}{2} e^{2}\right)\right. \\
& \left.-\frac{1}{2}\left(1-e+\frac{5}{2} e^{2}\right) \sin ^{2} I\right] \\
& -\frac{15}{8} \frac{a^{4} a^{\prime 2}}{A^{6}}\left(1+\frac{7}{2} \frac{\rho}{A^{2}}\right)\left[\left(1+5 e^{2}+\frac{5}{16} e^{4}\right)\right. \\
& \left.+\left(-\frac{1}{2}+\frac{1}{2} e-3 e^{2}+\frac{5}{8} e^{4}\right) \sin ^{2} I\right] \\
& +\frac{105}{32} \frac{a^{6} a^{2}}{A^{8}}\left[\left(1+\frac{21}{2} e^{2}+\frac{105}{8} e^{4}+\frac{35}{16} e^{6}\right)\right. \\
& \left.-\frac{1}{2}\left(1+\frac{21}{2} e^{2}+\frac{105}{8} e^{4}+\frac{35}{16} e^{6}\right) \sin ^{2} I\right] \\
& +\frac{105}{64} \frac{a^{4} a^{\prime 4}}{A^{8}}\left[\left(1+5 e^{2}+\frac{5}{16} e^{4}\right)-\left(\frac{3}{4}+\frac{81}{16} e^{2}+\frac{1}{2} e^{4}\right) \sin ^{2} I\right. \\
& \left.\left.-\frac{1}{2}\left(1-\frac{1}{4} e^{2}-\frac{3}{4} e^{4}\right) \sin ^{4} I\right]\right\} \\
& +\frac{\mu^{\prime}}{A}\left\{\frac{3}{4} \frac{a^{2} a^{\prime 2}}{A^{4}}\left(1+\frac{5}{2} \frac{\rho}{A^{2}}+\frac{35}{8} \frac{\rho^{2}}{A^{4}}\right) \frac{e}{2}\left(1+\frac{3}{2} e\right) \sin ^{2} I\right. \\
& -\frac{15}{8} \frac{a^{4} a^{\prime 4}}{A^{6}}\left(1+\frac{7}{2} \frac{\rho}{A^{2}}\right) \frac{e}{2}\left(1+\frac{17}{4} e+\frac{21}{8} e^{3}\right) \sin ^{2} I
\end{aligned}
$$




$$
\begin{aligned}
& -\frac{105}{32} \frac{a^{6} a^{\prime 2}}{A^{8}}\left(1+6 e^{2}+\frac{45}{8} e^{4}+\frac{25}{32} e^{6}\right) \sin ^{2} I \\
& +\frac{105}{64} \frac{a^{4} a^{\prime 4}}{A^{8}}\left[\frac{1}{2}\left(1+5 e^{2}+\frac{5}{16} e^{4}\right)+\left(-\frac{3}{2}+3 e^{2}+\frac{53}{32} e^{4}\right) \sin ^{2} I\right. \\
& \left.\left.+\left(1-\frac{1}{4} e^{2}-\frac{3}{4} e^{4}\right) \sin ^{4} I\right]\right\} \cos 2 \omega \\
& +\frac{\mu^{\prime}}{A}\left\{\frac { 1 0 5 } { 6 4 } \frac { a ^ { 4 } a ^ { \prime 4 } } { A ^ { 8 } } \left[\frac{1}{2}\left(1-\frac{23}{8} e^{2}+\frac{15}{8} e^{4}\right)\right.\right. \\
& \left.\left.-\frac{1}{2}\left(1-\frac{1}{4} e^{2}-\frac{3}{4} e^{4}\right) \sin ^{2} I\right] \sin ^{2} I\right\} \cos 4 \omega .
\end{aligned}
$$

For the specific case of Encke's Comet, assuming

$$
\begin{aligned}
a^{\prime} & =5.2028 \mathrm{AU} \text { (Jupiter) } \\
a & =2.217 \mathrm{AU} \\
e & =0.847 \\
\mu^{\prime} & =G m^{\prime}=0.28253 \times 10^{-6}(\mathrm{AU})^{3} \mathrm{~d}^{-2} \\
I & =13.9,
\end{aligned}
$$

it follows that the basic parameters of the problem have the values

$$
\begin{aligned}
A & =9.2976 \mathrm{AU} \\
\rho & =-25.8374(\mathrm{AU})^{2} \\
\rho / A^{2} & =-0.29889 \\
\mu^{\prime} / A & =0.30388 \times 10^{-7}(\mathrm{AU})^{2} \mathrm{~d}^{-2} \\
a / A & =0.23845 \\
a^{\prime} / A & =0.55959 \\
\sin I & =0.2402 \\
\cos I & =0.9707 .
\end{aligned}
$$

The maximum powers kept in the previous development correspond to reasonably small values:

$$
\begin{aligned}
(a / A)^{8} & =1.0451 \times 10^{-5} \\
\left(a^{3} a^{\prime} / A^{4}\right)^{2} & =5.7561 \times 10^{-5} \\
\left(a a^{\prime} / A^{2}\right)^{4} & =3.1698 \times 10^{-4},
\end{aligned}
$$

as compared with the main term, which is equal to unity. Of course, the real advantage of the new method is for orbits of high eccentricity which lie partly inside and partly 
outside the orbit of a 'primary'. The only method available up to now to solve this problem was that of 'partial anomalies' introduced by Hansen.

\section{Variation of Elements: Many Planets}

The equations for the long-period and secular variations of the elements to be considered are

$$
\begin{aligned}
& I=\frac{\cot I}{n a^{2}\left(1-e^{2}\right)^{1 / 2}} \frac{\partial R}{\partial \omega} \\
& \dot{\omega}=-\frac{\cot I}{n a^{2}\left(1-e^{2}\right)^{1 / 2}} \frac{\partial R}{\partial I}+\frac{\left(1-e^{2}\right)^{1 / 2}}{n a^{2} e} \frac{\partial R}{\partial e},
\end{aligned}
$$

while $e$ is obtained through the integral $\sqrt{ }\left(1-e^{2}\right) \cos I=\hat{C}$, and $l$ and $\Omega$ can be obtained by direct integration of the respective equations - since they are not present in $R$. The disturbing function $R$ is defined by the secular part of $U$, for all planets under consideration, so that it may be written

$$
R=\sum_{i=1}^{N} G m A_{i} \sum_{j=0}^{J} M_{i j}\left(e, I, a_{i}^{\prime}, a, \rho_{i}, A_{\imath}\right) \cos 2 j \omega,
$$

where $i$ refers to the particular planet, $N$ is the number of planets considered and the maximum value $J$ of $j$ is $2[k / 2]$, twice the integer part of half the maximum power reached by $A_{i}^{-1}$ in the development. Evidently this maximum power does not need to be the same for all planets. Also, in this simple formulation, we have assumed all planets in the same plane, a condition which can easily be removed.

Introducing $\hat{C}$ instead of $e$, we find

$$
\begin{aligned}
& I=\frac{\cos ^{2} I}{\sin I} \frac{1}{\hat{C} n a^{2}} \frac{\partial R}{\partial \omega} \\
& \dot{\omega}=-\frac{\cos ^{2} I}{\sin I} \frac{1}{\hat{C} n a^{2}} \frac{\partial R}{\partial I}+\frac{\hat{C}}{n a^{2} \cos I} \frac{1}{e} \frac{\partial R}{\partial e},
\end{aligned}
$$

where, after differentiation, $e$ has to be substituted by

$$
e=\left\{1-\hat{C}^{2} \sec ^{2} I\right\}^{1 / 2} .
$$

The geometrical behavior of the orbit can be obtained by considering the fact that $R$, as a function of $e$ and $\omega$ with parameters $\hat{C}$ and $a$, is constant along an orbit (except for short periodic variations), so that one can find $R=$ const. curves in the $(\omega, e)$ plane. The time dependence is obtained by integrating the above system, and owing to the very complex form of $R$ this is, in general, best done numerically.

\section{The Eccentricities of the Planets}

It has been shown by several authors that the eccentricity of the orbit of the planet plays an important role in the long-term behavior of cometary orbits. In order to 
modify the disturbing function accordingly, it is necessary to substitute $r^{\prime}$ for $a^{\prime}$; that is, the ring has to be considered elliptical. Again, one can proceed by considering the identity

$$
\Delta^{2}=A^{2}-\left(A^{2}-\Delta^{2}\right)
$$

where now

and

$$
A=a(1+e)+a^{\prime}\left(1+e^{\prime}\right)
$$

$$
\cos \psi=\cos \delta \cos \left(\alpha^{\prime}-\alpha\right),
$$

with the difference that now the integral over the ring is not circular but elliptic. Since

$$
\Delta^{2}=r^{2}+r^{2}-2 r r^{\prime} \cos \psi
$$

it follows that

$$
\begin{aligned}
\Delta^{-1}= & A^{-1} \sum_{k=0}^{\infty} \sum_{j=0}^{k} \sum_{v=0}^{j} P_{k j p} A^{-2 k}\left(A^{2}-r^{2}\right)^{k-j} \cos ^{p} \delta \\
& \times r^{\prime 2 j-p} \cos ^{p}\left(\alpha^{\prime}-\alpha\right),
\end{aligned}
$$

where

$$
\alpha^{\prime}-\alpha=f^{\prime}-\theta,
$$

$f^{\prime}$ being the true anomaly of the point $P^{\prime}$ on the ring,

$$
\theta=\Omega-\omega^{\prime}+u,
$$

with $\omega^{\prime}$ the longitude of perihelion of the ring and $u=\alpha-\Omega$,

$$
r^{\prime}=\frac{a^{\prime}\left(1-e^{\prime 2}\right)}{1+e^{\prime} \cos f^{\prime}},
$$

and

$$
P_{k j p}=\left(\begin{array}{l}
k \\
j
\end{array}\right)\left(\begin{array}{l}
j \\
p
\end{array}\right)(-2)^{p}\left(\begin{array}{c}
-\frac{1}{2} \\
k
\end{array}\right)(-1)^{k} .
$$

The integral over the elliptic ring can be performed using the eccentric anomaly $E^{\prime}$; that is, introducing the transformation

$$
\frac{\mathrm{d} f^{\prime}}{\mathrm{d} E^{\prime}}=\frac{a^{\prime} \sqrt{1-e^{\prime 2}}}{r^{\prime}},
$$

so that, for $j \neq 0$,

$$
\begin{aligned}
I_{j p} & =\frac{1}{2 \pi} \int_{0}^{2 \pi} r^{2 j-p} \cos ^{p}\left(f^{\prime}-\theta\right) \mathrm{d} f^{\prime} \\
& =\frac{a^{\prime} \sqrt{1-e^{\prime 2}}}{2 \pi} \int_{0}^{2 \pi} r^{\prime 2 j}{ }^{p-1} \cos ^{p}\left(f^{\prime}-\theta\right) \mathrm{d} E^{\prime} .
\end{aligned}
$$


On the other hand,

$$
\cos ^{p}\left(f^{\prime}-\theta\right)=\sum_{q=0}^{p}\left(\begin{array}{l}
p \\
q
\end{array}\right) \cos ^{q} \theta \sin ^{p-q} \theta \cos ^{q} f^{\prime} \sin ^{p-q} f^{\prime}
$$

and therefore

$$
\begin{aligned}
I_{j p}= & \frac{a^{\prime} \sqrt{1-e^{\prime 2}}}{2 \pi} \sum_{q=0}^{p}\left(\begin{array}{l}
p \\
q
\end{array}\right) \cos ^{q} \theta \sin ^{p-q} \theta \\
& \times \int_{0}^{2 \pi} x^{\prime q} y^{\prime p-q} r^{\prime 2 j-2 p-1} \mathrm{~d} E^{\prime} .
\end{aligned}
$$

The integration can be easily performed for $j \neq 0, p=0,1, \ldots, j-1$, for in this case $2 j-2 p-1>0$.

For $j=0(p=0)$,

$$
I_{00}=\frac{1}{2 \pi} \int_{0}^{2 \pi} \mathrm{d} f^{\prime}=1 .
$$

For $j \neq 0, p=j$,

$$
\frac{1}{2 \pi} \int_{0}^{2 \pi} x^{\prime q} y^{j-q} \frac{\mathrm{d} E^{\prime}}{r^{\prime}}=\frac{a^{\prime j-1} \eta^{j-q}}{2 \pi} \int_{0}^{2 \pi} \frac{\left(\cos E^{\prime}-e^{\prime}\right)^{q} \sin ^{j-q} E^{\prime}}{1-e^{\prime} \cos E^{\prime}} \mathrm{d} E^{\prime},
$$

which is zero for $j-q$ odd. For $j-q$ even, let $j-q=2 s$. Then, since

$$
\begin{aligned}
\cos ^{p} x & =2^{1-p} \sum_{n=0}^{[k / 2]}\left(\begin{array}{l}
p \\
n
\end{array}\right) \varepsilon_{n} \cos [(p-2 n) x] \\
\varepsilon_{n} & =1 \quad(p \text { odd }) \\
\varepsilon_{n} & =1, \quad n=0,1, \ldots, \frac{p}{2}-1 \quad(p \text { even }) \\
\varepsilon_{p / 2} & =\frac{1}{2} \quad(p \text { even })
\end{aligned}
$$

and

$$
\frac{1}{2 \pi} \int_{0}^{2 \pi} \frac{\cos n x}{1-e \cos x} \mathrm{~d} x=\frac{\left(\sqrt{1-e^{2}}-1\right)^{n}}{(-e)^{n} \sqrt{ }\left(1-e^{2}\right)}
$$

we obtain

$$
\begin{aligned}
\frac{1}{2 \pi} \int_{0}^{2 \pi} \frac{\left(\cos E^{\prime}-e^{\prime}\right)^{q} \sin ^{j-q} E^{\prime}}{1-e^{\prime} \cos E^{\prime}} \mathrm{d} E^{\prime} \\
=\sum_{\alpha=0}^{q} \sum_{\beta=0}^{s} \sum_{\gamma=0}^{[(\alpha+2 \beta) / 2]}\left(\begin{array}{l}
q \\
\alpha
\end{array}\right)\left(\begin{array}{l}
s \\
\beta
\end{array}\right)\left(\begin{array}{c}
\alpha+2 \beta \\
\gamma
\end{array}\right) \varepsilon_{\gamma}(-1)^{q+\beta-\alpha} 2^{1-\alpha-2 \beta} \\
\quad \times \eta^{\prime-1} e^{\prime q-2 \alpha-2 \beta+2 \gamma}\left(\eta^{\prime}-1\right)^{\alpha+2 \beta-2 \gamma},
\end{aligned}
$$


76

G. E. O. GiaCAGLiA

so that

$$
\begin{aligned}
I_{j j}= & a^{\prime j} \sum_{s=0}^{[j / 2]}\left(\begin{array}{c}
j \\
j-2 s
\end{array}\right) \cos ^{j-2 s} \theta \sin ^{2 s} \theta \eta^{\prime 2 s} \\
& \times \sum_{\alpha=0}^{j-2 s} \sum_{\beta=0}^{s} \sum_{\gamma}^{[(\alpha+2 \beta) / 2]}\left(\begin{array}{c}
j-2 s \\
\alpha
\end{array}\right)\left(\begin{array}{l}
s \\
\beta
\end{array}\right)\left(\begin{array}{c}
\alpha+2 \beta \\
\gamma
\end{array}\right) \varepsilon_{\gamma}(-1)^{j+\beta} 2^{1-\alpha-2 \beta} \\
& \times e^{j-2(s+\alpha+\beta-\gamma)}\left(1-\eta^{\prime}\right)^{\alpha+2(\beta-\gamma)} .
\end{aligned}
$$

For $j \neq 0, p=0,1, \ldots, j-1$,

$$
\begin{aligned}
I_{j p}= & {a^{\prime 2 j-p}}^{\prime} \sum_{q=0}^{p}\left(\begin{array}{l}
p \\
q
\end{array}\right) \cos ^{q} \theta \sin ^{p-q} \theta \eta^{\prime p-q} \\
& \times \frac{1}{2 \pi} \int_{0}^{2 \pi}\left(\cos E^{\prime}-e^{\prime}\right)^{q} \sin ^{p-q} E^{\prime}\left(1-e^{\prime} \cos E^{\prime}\right)^{2 j-2 p-1} \mathrm{~d} E^{\prime} .
\end{aligned}
$$

Again, this is zero if $p-q$ is odd, so that, setting $p-q=2 s$,

$$
\begin{aligned}
I_{j p}= & a^{\prime 2 j-p} \eta^{\prime} \sum_{s=0}^{[k / 2]}\left(\begin{array}{c}
p \\
p-2 s
\end{array}\right) \cos ^{p-2 s} \theta \sin ^{2 s} \theta \eta^{\prime 2 s} \\
& \times \frac{1}{2 \pi} \int_{0}^{2 \pi}\left(\cos E^{\prime}-e^{\prime}\right)^{p-2 s}\left(1-e^{\prime} \cos E^{\prime}\right)^{2 j-2 p-1}\left(1-\cos ^{2} E^{\prime}\right)^{s} \mathrm{~d} E^{\prime},
\end{aligned}
$$

which we write as

$$
\begin{aligned}
I_{j p}= & a^{22-p} \sum_{s=0}^{[k / 2]} G_{j p s}\left(e^{\prime}\right) \cos ^{p-2 s} \theta \sin ^{2 s} \theta \\
G_{j j s}= & \left(\begin{array}{c}
j \\
j-2 s
\end{array}\right) \eta^{\prime 2 s} \sum_{s=0}^{j-2 s} \sum_{\beta=0}^{s} \sum_{\gamma=0}^{[\alpha / 2]+\beta}\left(\begin{array}{c}
j-2 s \\
\alpha
\end{array}\right)\left(\begin{array}{c}
s \\
\beta
\end{array}\right)\left(\begin{array}{c}
\alpha+2 \beta \\
\gamma
\end{array}\right) \varepsilon_{\gamma} \\
& \times(-1)^{j+\beta} 2^{1-\alpha-2 \beta} e^{j-2(s+\alpha+\beta-\gamma)}\left(1-\eta^{\prime}\right)^{\alpha+2(\beta-\gamma)} \\
G_{000}= & 1,
\end{aligned}
$$

and for $p \neq j(j \neq 0)$,

$$
\begin{aligned}
G_{j p s}= & \eta^{\prime 2 s+1}\left(\begin{array}{c}
p \\
p-2 s
\end{array}\right) \\
& \times \frac{1}{2 \pi} \int_{0}^{2 \pi}\left(\cos E^{\prime}-e^{\prime}\right)^{p-2 s}\left(1-e^{\prime} \cos E^{\prime}\right)^{2 j-2 p-1}\left(1-\cos ^{2} E^{\prime}\right)^{s} \mathrm{~d} E^{\prime} \\
= & \left(\begin{array}{c}
p \\
p-2 s
\end{array}\right) \eta^{\prime 2 s+1} \sum_{\alpha=0}^{p-2 s} \sum_{\gamma=0}^{s} \sum_{\beta=[(\alpha+1) / 2]}^{[(\alpha-1) / 2]+j-p}\left(\begin{array}{c}
p-2 s \\
\alpha
\end{array}\right)\left(\begin{array}{c}
2 j-2 p-1 \\
2 \beta-\alpha
\end{array}\right) \\
& \times\left(\begin{array}{l}
s \\
\gamma
\end{array}\right)(-1)^{\gamma+\beta+p} \frac{(2 \beta+2 \gamma-1) ! !}{2^{\beta+\gamma}(\beta+\gamma) !} e^{{ }^{k+p-2(\alpha+s)}}
\end{aligned}
$$

https://doi.org/10.1017/S007418090000629X Published online by Cambridge University Press 
We thus arrive at the force function for the elliptical ring:

$$
\begin{aligned}
U= & \frac{G m^{\prime}}{A} \sum_{k=0}^{\infty} \sum_{j=0}^{k} \sum_{p=0}^{j} P_{k j p} A^{-2 k}\left(A^{2}-r^{2}\right)^{k-j} \cos ^{p} \delta \\
& \times a^{2 j-p} \sum_{s=0}^{[p / 2]} G_{j p s}\left(e^{\prime}\right) \cos ^{p-2 s} \theta \sin ^{2 s} \theta .
\end{aligned}
$$

But now,

$$
\theta=\left(\Omega-\omega^{\prime}\right)+u
$$

where

$$
\begin{aligned}
& \cos \delta \cos u=\cos (\omega+f) \\
& \cos \delta \sin u=\sin (\omega+f) \cos I .
\end{aligned}
$$

We have that

$$
\begin{aligned}
\cos ^{p} \delta \cos ^{p-2 s} \theta \sin ^{2 s} \theta & \\
= & \sum_{\alpha=0}^{p-2 s} \sum_{q=\alpha}^{\alpha+2 s}\left(\begin{array}{c}
p-2 s \\
\alpha
\end{array}\right)\left(\begin{array}{c}
2 s \\
q-\alpha
\end{array}\right)(-1)^{\alpha} \cos ^{p+4-2(s+\alpha)}\left(\Omega-\omega^{\prime}\right) \\
& \times \sin ^{2(s+\alpha)-q}\left(\Omega-\omega^{\prime}\right) \cos ^{p-q} \delta \cos ^{p-q} u \cos ^{q} \delta \sin ^{q} u,
\end{aligned}
$$

and therefore

$$
\begin{aligned}
\cos ^{p} \delta \cos ^{p-2 s} \theta \sin ^{2 s} \theta= & \sum_{\alpha=0}^{p-2 s} \sum_{q=\alpha}^{\alpha+2 s}\left(\begin{array}{c}
p-2 s \\
\alpha
\end{array}\right)\left(\begin{array}{c}
2 s \\
q-\alpha
\end{array}\right)(-1)^{\alpha} \\
& \times \cos ^{p+q-2(s+\alpha)}\left(\Omega-\omega^{\prime}\right) \sin ^{2(s+\alpha)-q}\left(\Omega-\omega^{\prime}\right) \\
& \times \cos ^{q} I \cos ^{p-q}(\omega+f) \sin ^{q}(\omega+f) .
\end{aligned}
$$

The contribution to the secular part of $U$ comes evidently from even values of $q$, so that we keep only those by writing

$$
\begin{aligned}
& {\left[\cos ^{p} \delta \cos ^{p-2 s} \theta \sin ^{2 s} \theta\right]_{\mathrm{even}}} \\
& =\sum_{\alpha=0}^{p-2 s} \sum_{q=[(\alpha+1) / 2]}^{[(\alpha+2 s) / 2]}\left(\begin{array}{c}
p-2 s \\
\alpha
\end{array}\right)\left(\begin{array}{c}
2 s \\
2 q-\alpha
\end{array}\right)(-1)^{\alpha} \cos ^{p+2(q-s-\alpha)}\left(\Omega-\omega^{\prime}\right) \\
& \quad \times \sin ^{2(s+\alpha-q)}\left(\Omega-\omega^{\prime}\right) \cos ^{2 q} I \cos ^{p-2 q}(\omega+f) \sin ^{2 q}(\omega+f) .
\end{aligned}
$$

On the other hand,

$$
\cos ^{p-2 q}(\omega+f) \sin ^{2 q}(\omega+f)=\sum_{\beta=0}^{q}\left(\begin{array}{l}
q \\
\beta
\end{array}\right)(-1)^{q-\beta} \cos ^{p-2 \beta}(\omega+f)
$$

and

$$
\left(A^{2}-r^{2}\right)^{k-j}=\sum_{\gamma=0}^{k-j} A^{2(k-j-\gamma)}\left(\begin{array}{c}
k-j \\
\gamma
\end{array}\right)(-1)^{y} r^{2 \gamma}
$$


so that, defining as before

$$
Q_{2 \gamma, p-2 \beta}=\frac{1}{2 \pi} \int_{0}^{2 \pi} r^{2 \gamma} \cos ^{p-2 \beta}(\omega+f) \mathrm{d} l,
$$

we have, for an elliptic ring,

$$
\begin{aligned}
U_{s}= & \frac{G m^{\prime}}{A} \sum_{k=0}^{\infty} \sum_{j=0}^{k} \sum_{p=0}^{j} \sum_{s=0}^{[p / 2]} P_{k j p} A^{-2 k} a^{2 j-p} G_{i p s}\left(e^{\prime}\right) \\
& \times \sum_{\gamma=0}^{k-} A^{2(k-j-\gamma)}\left(\begin{array}{c}
k-j \\
\gamma
\end{array}\right)(-1)^{\gamma} \sum_{\alpha=0}^{p-2 s} \sum_{q=[(\alpha+1) / 2]}^{[(\alpha+2 s) / 2]}\left(\begin{array}{c}
p-2 s \\
\alpha
\end{array}\right)\left(\begin{array}{c}
2 s \\
2 q-\alpha
\end{array}\right) \\
& \times(-1)^{\alpha} \cos ^{2 q} I \cos ^{p+2(q-s-\alpha)}\left(\Omega-\omega^{\prime}\right) \sin ^{2(s+\alpha-q)}\left(\Omega-\omega^{\prime}\right) \\
& \times \sum_{\beta=0}^{q}\left(\begin{array}{l}
q \\
\beta
\end{array}\right)(-1)^{q-\beta} Q_{2 \gamma, p-2 \beta} .
\end{aligned}
$$

It remains to determine $Q_{2 \gamma, p-2 \beta}$ for $p$ odd, since for $p$ even $(=2 \nu)$ we have, from Equation (15),

$$
Q_{2 \gamma, 2(v-\beta)}=a^{2 \gamma} \sum_{\sigma=0}^{\nu-\beta} M_{2 \gamma, 2(v-\beta)}^{\sigma} \cos 2 \sigma \omega .
$$

Consider therefore $p=2 v+1$, then we require $Q_{2 \gamma, 2(v-\beta)+1}$; we obtain

$$
Q_{2 q, 2 s+1}=\frac{1}{2 \pi} \int_{0}^{2 \pi} r^{2 q} \cos ^{2 s+1}(\omega+f) \mathrm{d} l,
$$

assuming, as is actually the case, that $0 \leq s \leq q$. Again, we introduce the eccentric anomaly as independent variable so that

$$
Q_{2 q, 2 s+1}=\frac{a^{2 q}}{2 \pi} \int_{0}^{2 \pi}\left(\frac{r}{a}\right)^{2 q+1} \cos ^{2 s+1}(\omega+f) \mathrm{d} E .
$$

As in Equation (13), we obtain

$$
\begin{aligned}
\left(\frac{r}{a}\right)^{2 q+1} & \cos ^{2 s+1}(\omega+f) \\
= & \frac{1}{2^{2 s+1}} \sum_{j=0}^{2 s+1} \sum_{k=0}^{2 s+1-2 j} \sum_{\gamma=k}^{k+2 q+1} \sum_{\alpha=0}^{k} \sum_{\beta=0}^{\gamma-k} L_{j, k, \gamma, \alpha, \beta}^{2 q, 2 s+1} \\
& \times e^{2(s-k-j)+\gamma+1}(1-\eta)^{k-\alpha}(1+\eta)^{\alpha} \\
& \times \exp [-i(2 s+1-2 j) \omega] \varepsilon^{\gamma \cdot 2(\alpha+\beta)}
\end{aligned}
$$


Isolating the even values of $\gamma$,

$$
\begin{aligned}
{\left[\left(\frac{r}{a}\right)^{2 q+1}\right.} & \left.\cos ^{2 s+1}(f+\omega)\right] \text { even } \\
= & \frac{1}{2^{2 s+1}} \sum_{j=0}^{2 s+1} \sum_{k=0}^{2 s+1-2 j[(k+1+2 q) / 2]} \sum_{\gamma=[(k+1) / 2]}^{k} \\
& \times \sum_{\alpha=0}^{k} \sum_{\beta=0}^{2 \gamma-k} L_{j, k, 2 \gamma, \alpha, \beta}^{2 q, 2 s+1} e^{2(s-k-j+\gamma)+1}(1-\eta)^{k-\alpha}(1+\eta)^{\alpha} \\
& \times \exp [-i(2 s+1-2 j) \omega] \varepsilon^{2[\gamma-(\alpha+\beta)]} .
\end{aligned}
$$

Finally one finds

$$
Q_{2 q, 2 s+1}=a^{2 q} \sum_{v=1}^{s} M_{2 q, 2 s+1}^{v} \cos [(2 v+1) \omega]
$$

where

$$
\begin{aligned}
M_{2 q, 2 s+1}^{v}= & \frac{1}{2^{2 s}} \sum_{k=0}^{2 v+1} \sum_{\gamma=[(k+1) ; 2]}^{[(k+2 q+1) ; 2]} \sum_{\alpha=0}^{k} L_{s-v, k, 2 \gamma, \alpha, \gamma-\alpha}^{2 q, 2 s+1} \\
& \times e^{2(v-k+\gamma)+1}(1-\eta)^{k-\alpha}(1+\eta)^{\alpha},
\end{aligned}
$$

which shows that the definition of the coefficients $M$ is the same as in the previous (even) case; see Equation (16). As was to be expected, both even and odd multiples of $\omega$ are now present in the $U_{s}$, together with terms in $\Omega-\omega^{\prime}$. The general argument is of the form

$$
2 k \omega+j\left(\omega+\Omega-\omega^{\prime}\right),
$$

with $k, j$ any integers, positive, negative or zero. Evidently, the system has two degrees of freedom, and there is no simple way of describing its behavior except by numerical means. For a realistic model, the perihelion $\omega^{\prime}$ of the perturbing planet should not be constant but move according to the theory of planetary secular perturbations.

\section{Conclusions}

The above derivations show that secular perturbations among bodies with arbitrary eccentricities and inclinations can be developed by analytical methods, provided that the possibility of collisions, or more realistically, of close approaches, is excluded. One can consider the secular perturbations by all the planets on the motion of a comet. An approximate calculation for the perturbations by the Earth on P/Encke shows that in order to keep terms (in the disturbing function) of the order of 0.01 , it is necessary to manipulate series of about 400 terms. Of course, the motions of comets are also influenced by nongravitational forces, but since the precise form of these forces seems to be somewhat obscure, it is not practicable to include them in this analysis. 


\section{Acknowledgments}

This research was partially supported by Office of Naval Research Contract N0001467-A-0126-0013. We are grateful to P. E. Nacozy and B. G. Marsden for information and several discussions which added to the completeness of the work.

\section{References}

Brouwer, D.: 1947, Astron. J. 52, 190.

Whipple, F. L.: 1940, Proc. Am. Phil. Soc. 83, 711. 